\title{
Complémentarité ou concurrence des aéroports : l'exemple du Grand Sud-Est
}

Alain Bonnafous, Professeur

Alexis Giret ${ }^{1}$, Economiste au Conseil National Routier

Dans le cadre de la préparation des « schémas de service » prescrits par la Loi d’Orientation d'Aménagement et de Développement du Territoire, l'Etat s'est trouvé confronté à des problèmes complexes, s'agissant du transport aérien : comment décliner, dans ce secteur, une politique visant à utiliser du mieux possible les complémentarités de l'offre ? N’y a-t-il pas lieu d’aménager territorialement celle-ci ? Si cet aménagement paraît souhaitable, quel peut être le rôle de l’Etat dans un contexte libéralisé ?

Cette dernière question a pris d'autant plus d'importance que les exercices des schémas de service ont été entamés peu après l'achèvement, en avril 1997, du premier grand pas européen dans la politique de libéralisation. Le jeu des compagnies aériennes européennes étant désormais surplombé par le jeu de la concurrence, l'Etat ne pouvait plus prétendre dicter les dessertes que les compagnies doivent offrir au nom de l'aménagement du territoire et de l'intérêt collectif. Il s'est ainsi interrogé sur les possibilités d'un fonctionnement interrégional en réseau des activités aéroportuaires. Une mission a été commise pour cela ${ }^{2}$, dont l'objet était limité à une configuration géographique se prêtant $a$ priori à cet exercice puisqu'elle concernait le transport aérien dans le Grand Sud-Est.

Le Laboratoire d'Economie des Transports a été sollicité dans ce cadre pour apporter quelques éléments de réflexion. Cet article s'appuie très largement sur ce travail ${ }^{3}$ conduit en 1999 et 2000, ce qui explique que les informations statistiques traitées soient relatives à 1998 et que seules les informations à caractère non statistique aient été mises à jour.

Les aéroports dans le Grand Sud-Est sont nombreux, mais tous ne jouent pas le même rôle. Nous limiterons notre réflexion aux seules activités des quatre aéroports principaux que sont Lyon Saint-Exupéry, Marseille Provence, Montpellier Méditerranée et Nice Côte d'Azur. Nous nous proposons de préciser, dans un premier temps, les termes du problème par l'analyse statistique des trafics.

Les responsabilités aéroportuaires en province étant largement décentralisées, il convient de repérer les points de vue des multiples acteurs locaux et leurs stratégies de développement. Présentés dans un deuxième point, ces points de vue ont fait l'objet d'entretiens approfondis en 1998 et 1999 et ont été, pour la suite, complétés par le suivi de la presse spécialisée.

\footnotetext{
${ }^{1}$ Alexis Giret était allocataire de recherche au Laboratoire d’Economie des Transports lorsqu’il a participé aux recherches qui alimentent cet article.

${ }^{2}$ Elle a été confiée au Directeur régional de l’Equipement de la région Rhône-Alpes de l'époque, Patrice Raulin.

${ }^{3}$ Celui-ci a donné lieu à deux rapports successifs (phase 1 et phase 2) signalés dans la bibliographie.
} 


\section{L’ACTIVITE AERIENNE DES QUATRE PRINCIPAUX AEROPORTS}

Par leurs volumes, comme par leurs structures de trafic, les quatre aéroports majeurs du Grand Sud-Est, Lyon Saint-Exupéry, Marseille Provence, Montpellier Méditerranée et Nice Côte d'Azur, présentent des configurations fort différentes, en particulier quant à leurs évolutions.

\section{I.1. Chroniques contrastées et effet TGV}

Lyon Saint-Exupéry, Marseille Provence et Nice Côte d'Azur étaient à un niveau comparable d'activité avant 1981, légèrement moindre pour Lyon qui subissait déjà une forte concurrence ferroviaire sur la liaison avec Paris. A partir de 1981, Lyon Saint-Exupéry a dû affronter la concurrence du TGV ${ }^{4}$ et a subi un sérieux décrochage. Marseille Provence est resté jusqu'en 1986 au niveau de Nice Côte d'Azur qui, par la suite, a distancé ses deux rivaux. Avec la mise en service du TGV Méditerranée, l’aéroport de Marseille s’est vu rattrapé, dès 2001, par celui de Lyon.

En dépit d'un taux de croissance «à deux chiffres» sur l'ensemble de la période 5 , Montpellier Méditerranée reste à un niveau modeste dont nous verrons qu'il reflète des structures de trafic très différentes de celles des trois autres sites (fig. 1).

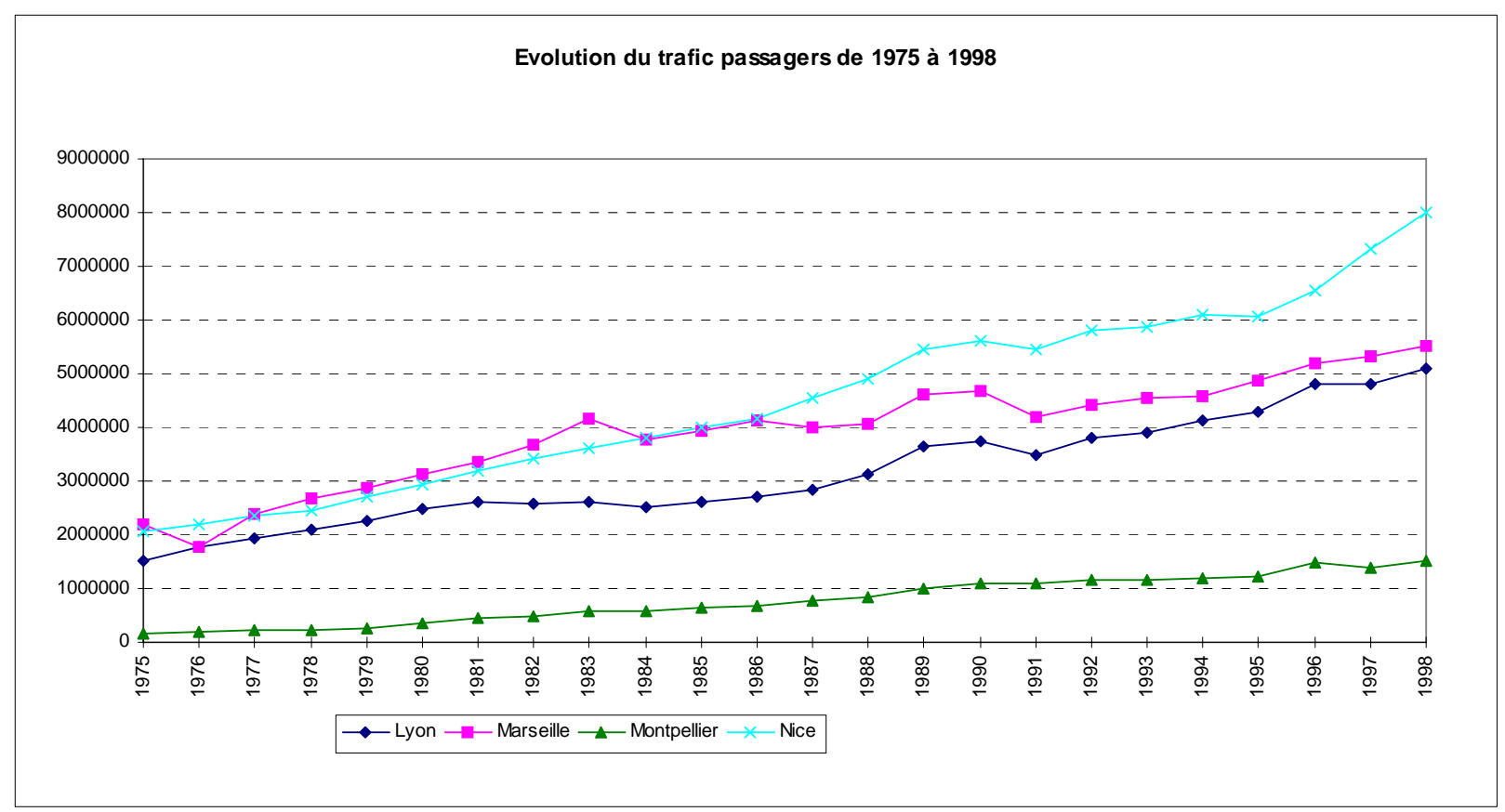

Figure 1 : Evolution du trafic passagers de 1975 à 1998

Source : DGAC 1998

Pour apprécier convenablement ces poids relatifs, il convient de les situer dans une perspective européenne.

\footnotetext{
${ }^{4}$ On peut résumer le choc concurrentiel en rappelant que la mise en service d'un premier tronçon de TGV mettait Lyon à trois heures de Paris en septembre 1981 et la mise en service complète à deux heures en septembre 1983.

${ }^{5}$ Entre 1975 et 1998, Montpellier a enregistré une croissance moyenne de 10,1\%, contre 6,1 \% pour Nice, 5,1\% pour Lyon et 4,1 \% pour Marseille.
} 


\section{I.2. Comparaisons européennes}

Nous ne considérerons pas seulement le poids des aéroports dans le tissu aéroportuaire européen mais aussi leur poids relatif par rapport à celui des leaders nationaux car ceci explique cela. Le tableau 1 donne le classement des 48 premiers aéroports européens suivant le nombre de passagers en 1997. La dernière colonne indique ce que représente le trafic des aéroports qui ne sont pas un leader national par rapport au trafic de celui-ci.

\begin{tabular}{|c|c|c|c|}
\hline Rang & Ville (plusieurs aéroports possibles) & Trafic passager 1997 & Trafic par rapport au premier aéroport national \\
\hline 1 & LONDON & 91135378 & \\
\hline 2 & PARIS & 60125194 & \\
\hline 3 & FRANKFURT & 39682996 & \\
\hline 4 & AMSTERDAM & 31021002 & \\
\hline 5 & ROMA & 25463570 & \\
\hline 6 & MADRID & 23121739 & \\
\hline 7 & ZURICH & 17870514 & \\
\hline 8 & MILANO & 17747188 & $70 \%$ \\
\hline 9 & MUNICH & 17625674 & $44 \%$ \\
\hline 10 & COPENHAGEN & 16607277 & \\
\hline 11 & PALMA DE MALLORCA & 16448610 & $71 \%$ \\
\hline 12 & STOCKHOLM & 15866453 & \\
\hline 13 & BRUSSELS & 15816357 & \\
\hline 14 & MANCHESTER & 15717489 & $17 \%$ \\
\hline 15 & DUSSELDORF & 15407407 & $39 \%$ \\
\hline 16 & BARCELONA & 14561994 & $63 \%$ \\
\hline 17 & MOSCOW & 14173950 & \\
\hline 18 & BERLIN & 11368162 & $29 \%$ \\
\hline 19 & ATHENS & 11090035 & \\
\hline 20 & DUBLIN & 10234638 & \\
\hline 21 & VIENNA & 9596577 & \\
\hline 22 & TENERIFE & 9480136 & $41 \%$ \\
\hline 23 & HAMBURG & 8546031 & $22 \%$ \\
\hline 24 & LAS PALMAS & 7926390 & $34 \%$ \\
\hline 25 & HELSINKI & 7678511 & \\
\hline 26 & NICE & 7305538 & $12 \%$ \\
\hline 27 & MALAGA & 7190651 & $31 \%$ \\
\hline 28 & STUTTGART & 6745403 & $17 \%$ \\
\hline 29 & LISBON & 6631472 & \\
\hline 30 & GLASGOW & 6581717 & $7 \%$ \\
\hline 31 & GENEVA & 5995126 & $34 \%$ \\
\hline 32 & BIRMINGHAM & 5909256 & $6 \%$ \\
\hline 33 & MARSEILLE & 5336348 & $9 \%$ \\
\hline 34 & COLOGNE & 5235945 & $13 \%$ \\
\hline 35 & LYON & 4818819 & $8 \%$ \\
\hline 36 & HANOVER & 4668135 & $12 \%$ \\
\hline 37 & ALICANTE & 4397931 & $19 \%$ \\
\hline 38 & TOULOUSE & 4299472 & $7 \%$ \\
\hline 39 & EDINBURGH & 4157829 & $5 \%$ \\
\hline 40 & PRAGUE & 4077707 & \\
\hline 41 & ARRECIFE & 4005103 & $17 \%$ \\
\hline 42 & FARO & 3663346 & \\
\hline 43 & BUDAPEST & 3619074 & \\
\hline 44 & WARSAW & 3547143 & \\
\hline 45 & IBIZA & 3528568 & $15 \%$ \\
\hline 46 & GOTEBORG & 3408168 & $21 \%$ \\
\hline 47 & LUTON & 3220995 & $4 \%$ \\
\hline 48 & NAPOLI & 3094146 & $12 \%$ \\
\hline$?$ & MONTPELLIER & 1413000 & $2 \%$ \\
\hline
\end{tabular}

Tableau 1 : Classement des 48 premiers aéroports européens suivant le nombre de passagers en 1997.

Source : ITA, tableau de bord 1997 
Hormis Paris, les aéroports français occupent des places bien modestes : entre la $26^{\text {ème }}$ et la $35^{\text {ème }}$ pour les trois premiers qui appartiennent tous trois au Grand Sud-Est, Montpellier étant, de loin, hors classement. Cette distribution des rôles entre la capitale et les métropoles régionales est un produit de l'histoire, qu'il s'agisse de la concentration sur Paris des fonctions internationales génératrices de trafic ou de l'organisation française de nos compagnies aériennes : à Air France les relations avec le reste du monde, hormis quelques relations réputées peu rentables avec les anciennes colonies ou les territoires d'outre-mer laissées à UTA ; à Air Inter les dessertes nationales, dont les plus lourdes sont des relations avec Paris.

Dans cette logique, Air France a pu apprécier très tôt les avantages des rendements croissants et le peu d'intérêt qu'il y aurait, pour la compagnie, à ouvrir trop de relations directes entre les places provinciales et l'étranger. D’une certaine manière, il s'agissait déjà, sans la désigner ainsi, d'une politique de hub et de gateway, observable partout où a dominé un opérateur historique national. Mais cela n'a pas partout hiérarchisé aussi sèchement le système. La figure 2 le montre clairement. Il concerne une sélection de pays de l'Union Européenne dont la superficie est comparable à celle de la France. Bien évidemment, certaines caractéristiques des territoires étudiés expliquent en partie ces résultats, telle l'insularité des Canaries, enregistrées au sein du réseau espagnol, ou la faible densité de population du territoire suédois.

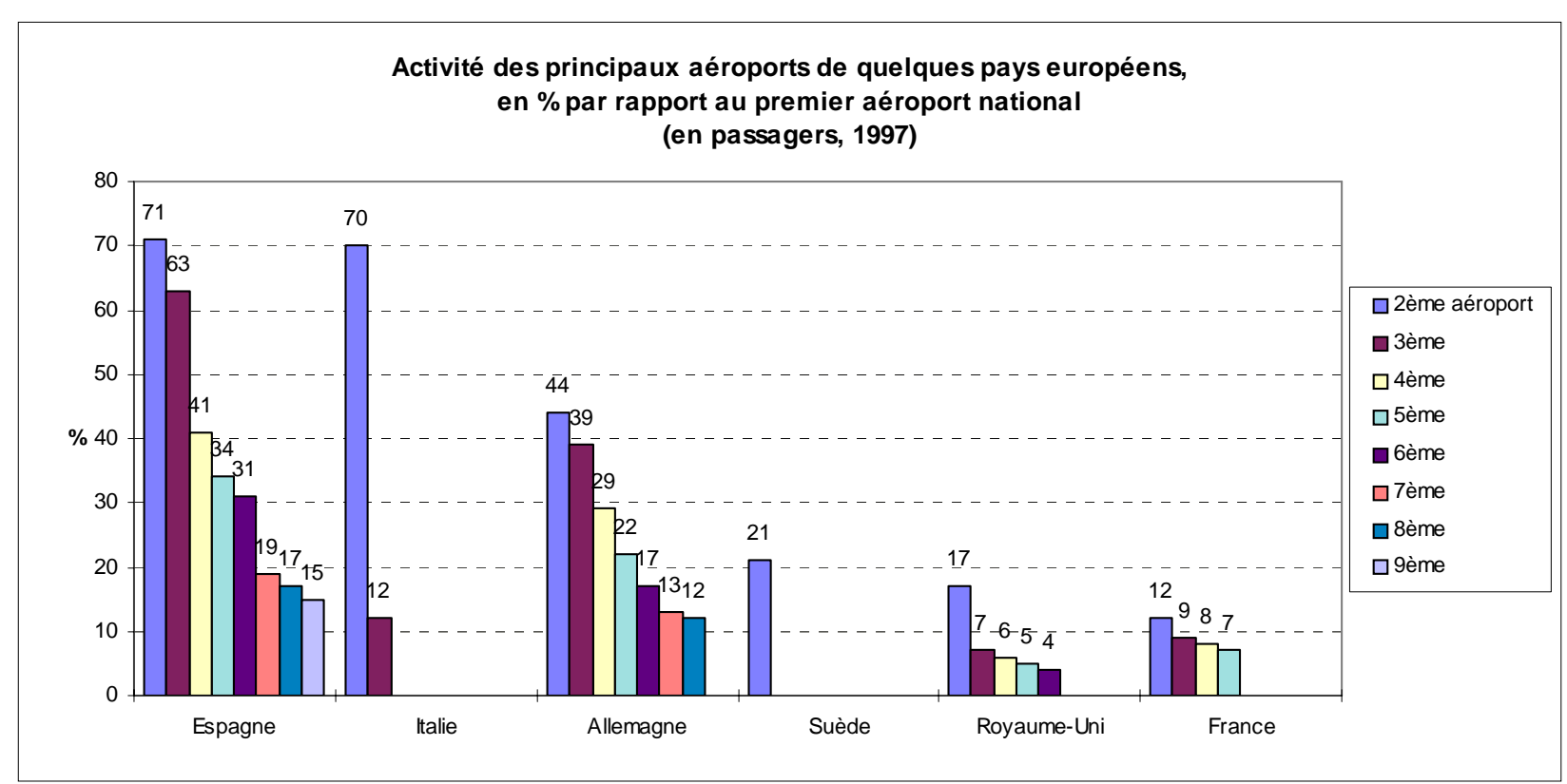

Figure 2 : Source : ITA, tableau de bord 1997

Plus généralement, on aperçoit clairement sur ce graphique les effets de la géographie et de l'histoire. On y retrouve, en particulier, la marque du « désert français », plus éloigné qu'il ne paraît d'un relatif désert britannique en dehors de Londres si l'on tient compte de l'étroitesse territoriale du Royaume-Uni. En revanche, la comparaison avec l'Allemagne est accablante pour nos aéroports de province : en nombre de passagers, la deuxième plate-forme française, Nice, est largement distancée par la cinquième plate-forme allemande qui est Hambourg.

De telles comparaisons ne relèvent pas d'un concours de " poids et haltères » entre aéroports. Elles désignent des enjeux très précis. Il y a, en effet, une corrélation forte entre la taille d'un aéroport, au sens traditionnel du nombre de passagers, et l'accessibilité que peut offrir cet aéroport à la région desservie. Cela correspond à une relation directe et toujours vérifiée entre 
le trafic et le nombre de dessertes offertes ${ }^{6}$, qui résulte lui même du nombre de destinations et des fréquences proposées. Le nombre de passagers n'est donc pas seulement une marque de puissance régionale, ni seulement le gage de recettes qui assurent le financement du développement d'un aéroport. Il est aussi un indicateur pertinent de l'accessibilité au reste du monde qu'il peut assurer.

Ces éléments de comparaison européenne suggèrent, en tout cas, que la notion française de métropoles régionales d'équilibre a un contenu encore bien modeste en matière de transports aériens.

\section{I.3. Spécificités géographiques des réseaux de desserte}

Parce qu'ils sont relativement faibles, les trafics assurés par les aéroports du Grand Sud-Est, ne peuvent concerner le monde entier. Ils sont, au contraire, marqués par des directions privilégiées, en somme des courants de trafics. Ils sont représentés, sur le graphique cidessous (figure 3), selon six grandes familles de destinations.

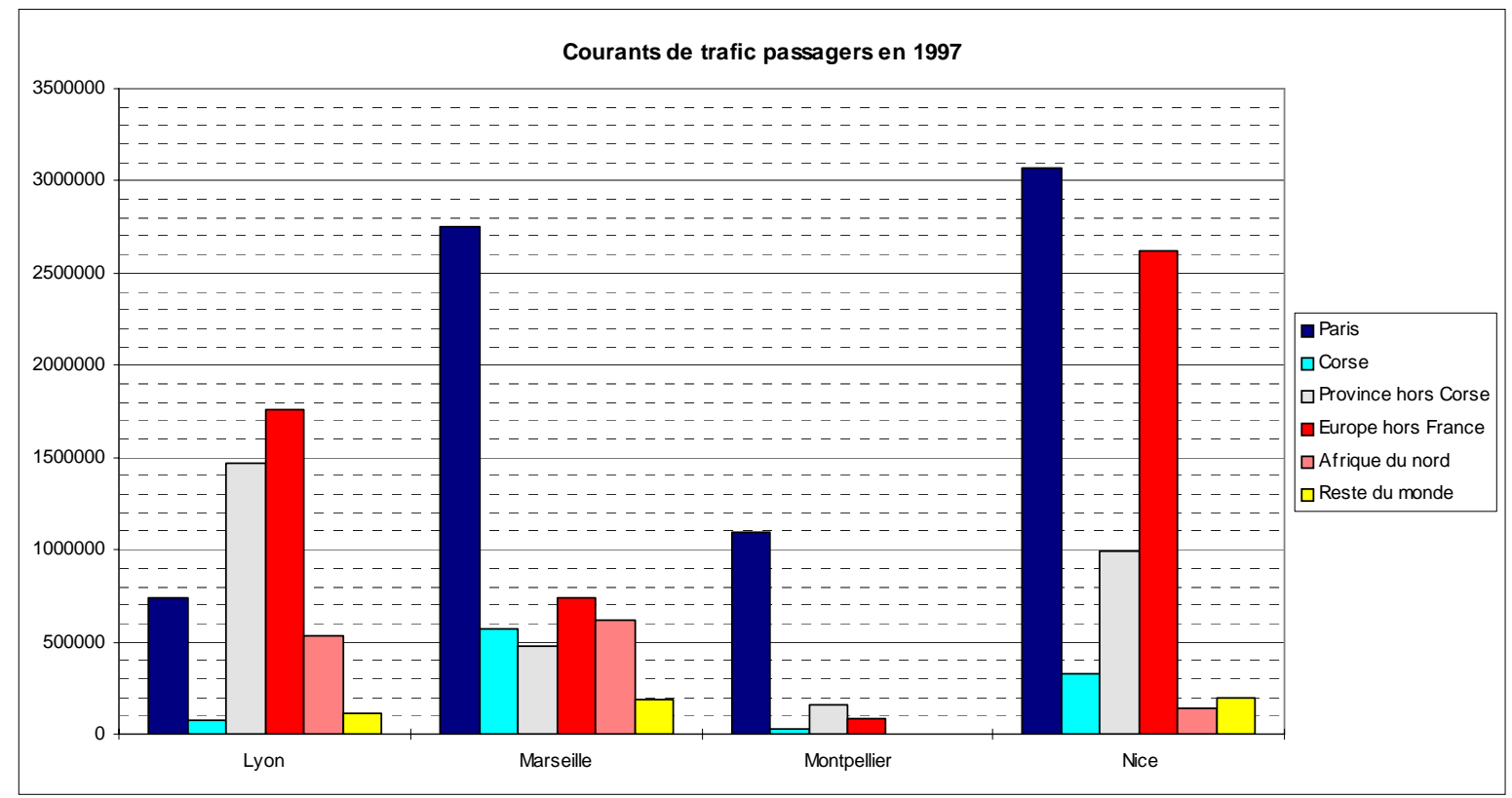

Figure 3 : courant de trafic passagers en 1997

Chacun des aéroports se distingue beaucoup plus clairement qu'avec les chiffres globaux: - Lyon Saint-Exupéry a un trafic avec Paris qui est le plus faible des quatre plates-formes. Cela tient évidemment à l'offre des services TGV entre Paris et Lyon qui sont à la fois plus fréquents, en moyenne tarifaire moins coûteux et plus rapides de centre à centre que les services aériens. Ceux-ci concernent, pour l'essentiel, des trajets en correspondance ou des destinations finales proches des aéroports de Paris. En revanche, Lyon Saint-Exupéry, à l'instar de Nice Côte d'Azur, a pu développer de très fortes activités avec la province et avec l'Europe.

- Marseille Provence est un aéroport marqué par son ouverture sur la Méditerranée et apparaît comme une entrée privilégiée depuis la Corse et l’Afrique du Nord.

- Hormis sa liaison avec Paris, Montpellier Méditerranée a des échanges faibles avec le reste de la France et plus faibles encore avec l'Europe.

- Nice Côte d'Azur apparaît comme l'aéroport le plus ouvert sur l'international, en particulier sur l'Europe.

\footnotetext{
${ }^{6}$ Des premiers essais de corrélation linéaire, avec différentes populations d’aéroports, conduisent à des rho toujours supérieurs à 0,87 et souvent supérieurs à 0,9.
} 
Cette décomposition par grandes destinations nous montre une autre conséquence de la grande concentration de nos trafics intercontinentaux sur les aéroports de Paris : le trafic longcourrier au départ des aéroports du Grand Sud-Est est faible. Il est partagé entre de rares lignes régulières (telle Nice - New-York à la date de notre observation statistique) et des vols charters.

L'analyse par destinations peut être encore quelque peu affinée en prenant en considération le détail des villes desservies et, pour chacune d'elle, les trafics concernés. L'importance des trafics est en effet une information sur la demande, mais elle est aussi un bon indicateur synthétique du service offert, en particulier en termes de fréquences. C’est ainsi que, dans ce qui suit, en ne considérant que les relations qui transportent plus de 50.000 passagers par an, nous sélectionnons aussi les destinations sur lesquelles l'offre est d'au moins un aller-retour quotidien dans chaque sens ${ }^{7}$.

Pour chacun des quatre aéroports, la première ville de destination en termes de trafic est Paris. Ayant déjà représenté ces trafics sur la précédente figure, nous ne les prenons pas en considération. La représentation de ce trafic dominant rendrait, du reste, les tracés moins lisibles. Ne figurent, en outre, que les lignes régulières inscrites sur les guides horaires "printemps-été » 1998. Les vols charters ou avec correspondance(s) sont exclus, à l'exception des vols avec un stop sans changement d'appareil qui se traduisent par un numéro de vol unique. Ces lignes sont représentées par des traits proportionnels au flux réalisés sur l’année entière 1998 (proportionnalité linéaire).

Dans le cas de Lyon, on peut observer des "rayons » relativement épais sur des relations nationales et vers les principales places européennes, notamment toutes celles qui proposent d'importantes dessertes intercontinentales. Cela peut refléter la position lyonnaise dans les trafics d'affaire, mais cette situation correspond aussi à ce qu'est devenu le « rendez-vous lyonnais » qui désigne une organisation des horaires, voulue par Air-France et conçue pour favoriser les correspondances du matin entre la première vague d'arrivées et la deuxième vague de départs, avec une offre symétrique en fin de journée ainsi qu'une offre de mijournée (15h-16h). Ce rendez-vous a été baptisé par la suite, d'une manière un peu pompeuse le deuxième hub français. Il supporte tout de même un système de correspondances qui alimente une part significative des trafics représentés sur ce graphique.

Les principaux trafics détaillés de Marseille Provence confirment l'orientation méditerranéenne : ces destinations pèsent plus, par exemple que les destinations européennes. Concernant l'accessibilité au reste du monde, la situation marseillaise est caractérisée par la faiblesse des accès à d'autres hubs majeurs que Londres et Paris (qui ne figure pas sur ce graphique). C'est ainsi que, contrairement à Lyon ou Nice, Marseille n’a pas d'accès régulier aux hubs de Francfort, de Zurich ou d'Amsterdam.

Montpellier Méditerranée propose une seule ligne, hors Paris, de plus de 50.000 passagers. A un niveau inférieur de trafic, une certaine diversité de lignes, essentiellement françaises, commence cependant à apparaître.

Nice Côte d'Azur propose le réseau international le plus complet. C'est ainsi que ses destinations régulières recouvrent pratiquement toutes celles de Lyon et Marseille en Europe. En revanche, Nice n’offre que quatre destinations significatives en province.

\footnotetext{
${ }^{7}$ Cela implique quatre vols sur 250 jours par an. Pour 50.000 passagers, chaque vol emporte alors en moyenne 50 passagers, ce qui correspond à des appareils de 60 à 70 places.
} 
Trafics de passagers de l'aéroport Lyon Saint-Exupéry Lignes régulières supérieures à 50000 passagers en 1998 (hors Paris)

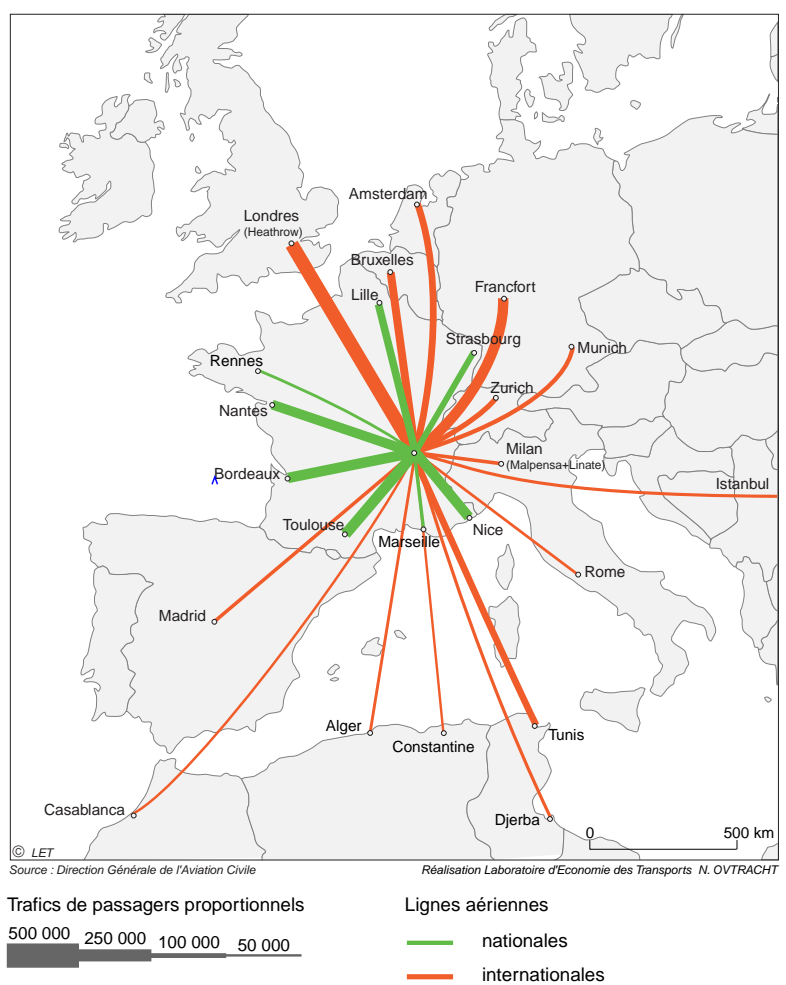

NB : Un trafic inclut les passagers origine et destination

Trafics de passagers de l'aéroport de Montpellier Méditerranée Lignes régulières supérieures à 50000 passagers en 1998 (hors Paris)

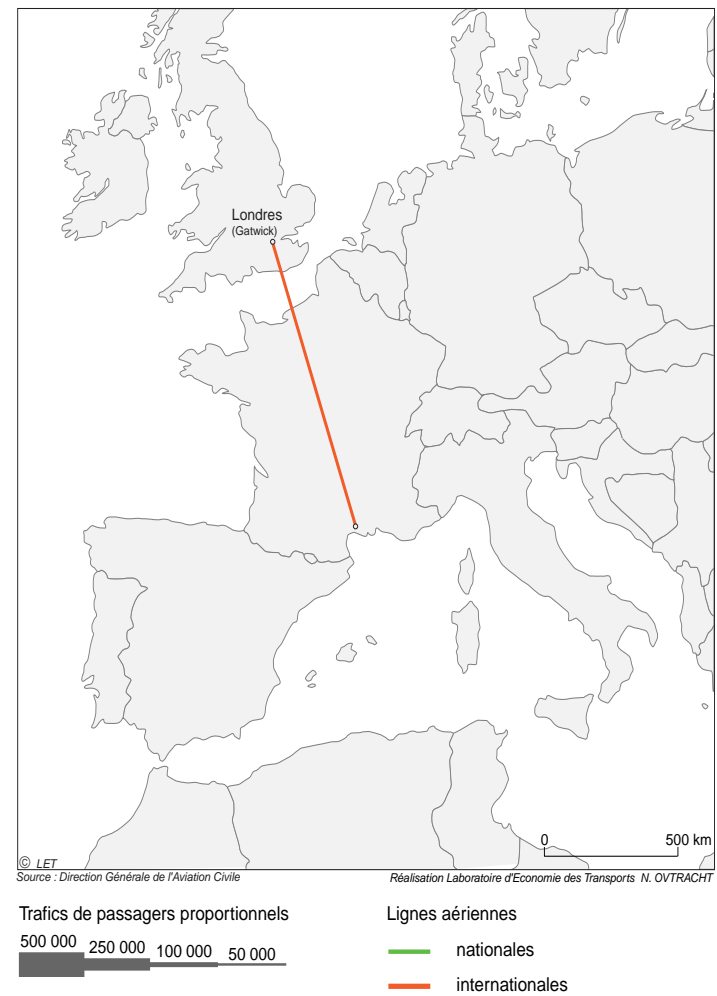

Trafics de passagers de l'aéroport Marseille Provence Lignes régulières supérieures à 50000 passagers en 1998 (hors Paris)

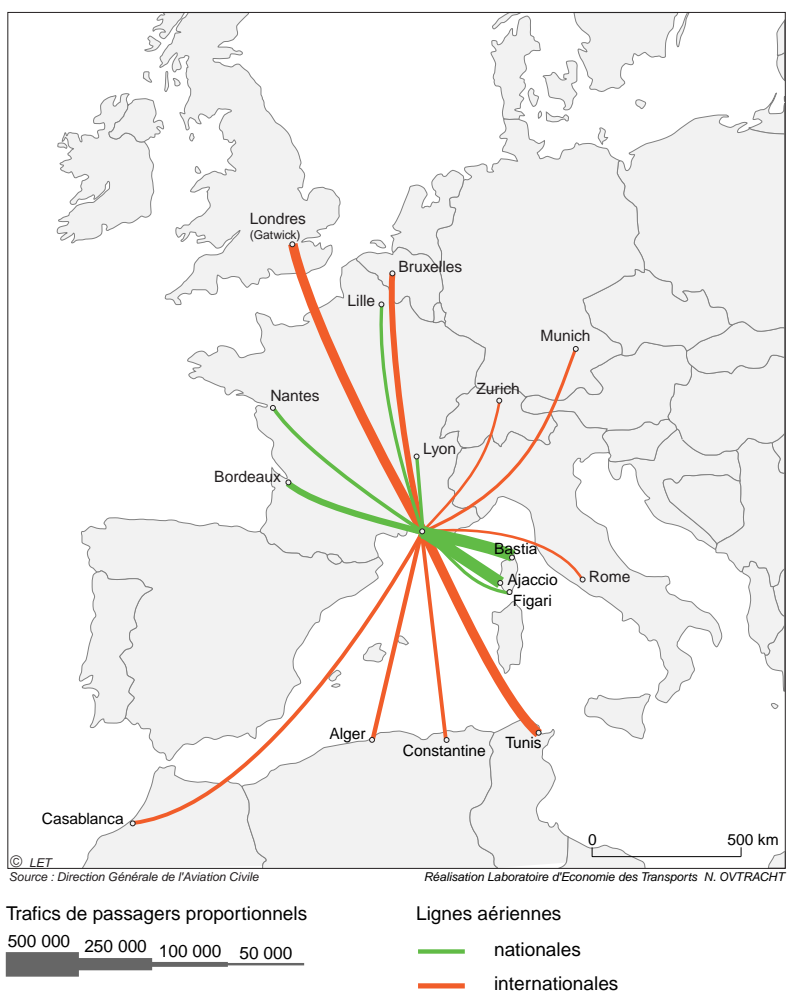

NB : Un trafic inclut les passagers origine et destination

Trafics de passagers de l'aéroport Nice Côte d'Azur Lignes régulières supérieures à 50000 passagers en 1998 (hors Paris)

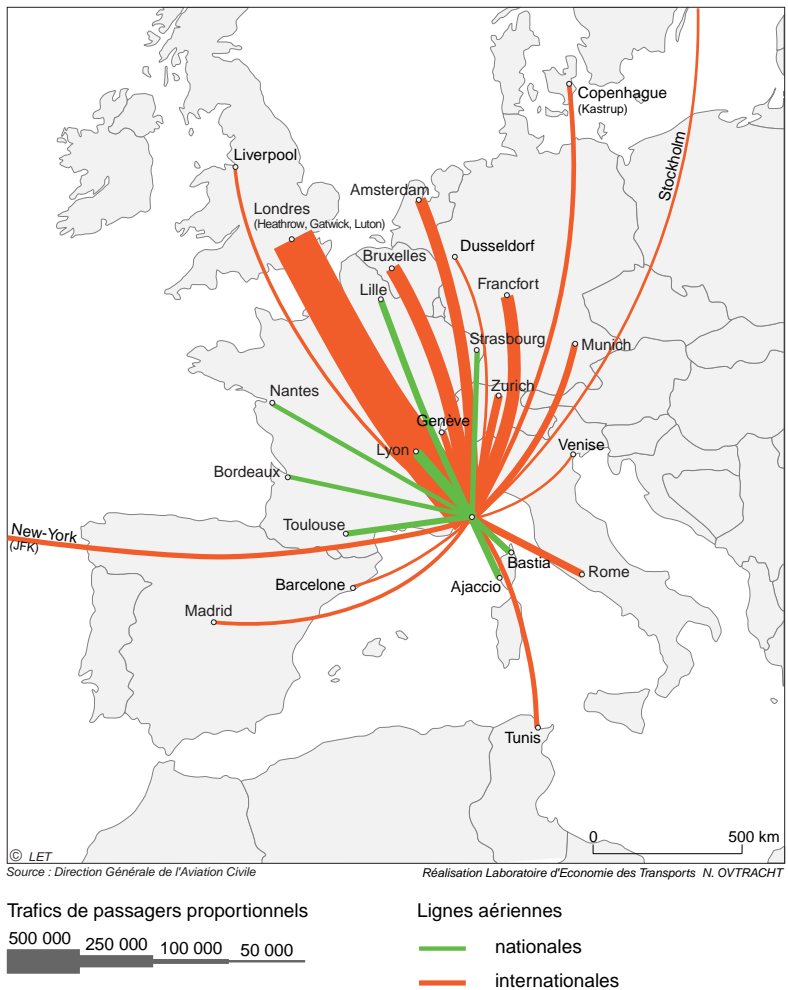

NB : Un trafic inclut les passagers origine et destination 
Ces observations en termes de trafics sont, en somme, assez cohérentes avec le jeu des acteurs que nous allons préciser dans une deuxième partie.

\section{LE JEU DES ACTEURS}

L'étude qui alimente cet article a donné lieu a de nombreux entretiens avec les acteurs concernés. Des responsables d'aéroports, de l'administration et de compagnies aériennes ont pu donner leurs sentiments sur les perspectives d'évolution des aéroports du Grand Sud-Est et sur la question de leur éventuelle complémentarité. Ce dernier terme a surpris beaucoup d'entre eux car il est tout bonnement étranger à leur positionnement, ainsi que l'illustrent quelques uns des propos qui ont été relevés dans les enquêtes.

\section{II.1. Des rôles bien distribués}

Les éléments dont nous rendrons rapidement compte sont significatifs de « l'après 1997 » et du système de régulation par la concurrence dont l’Europe a choisi de se doter.

\section{Les concessionnaires d'aéroports}

Un responsable de l'aéroport Nice Côte d'Azur a été un des rares a reconnaître cette notion de complémentarité : "A propos de la concurrence entre aéroports, nous ne la ressentons pas vraiment, nous constatons plus une complémentarité. Bien qu'il soit très fort sur le Maghreb, Marseille Provence a encore une dimension nationale. A côté de cela, Nice Côte d'Azur a acquis une importance européenne. Notre seul véritable lieu de concurrence est la Corse. Lyon Saint-Exupéry aussi a une taille européenne, mais, plus étonnant, on constate en général une complémentarité. Par exemple, les lignes récemment créées vers Varsovie et Lisbonne passent systématiquement par Lyon Saint-Exupéry et Nice Côte d'Azur. » Il cite avec ces deux destinations les rares cas concrets de complémentarité. Quant à celle avec Marseille, son évocation est surtout l'occasion de vanter l'avance de Nice en termes d'offre. En fait, l'isolement relatif de Nice, dans le Grand Sud-Est, l'éloigne de toute réelle concurrence de la part des autres grands aéroports de cette région. La problématique de Nice se pose autrement. « La vraie question se pose en ces termes : quel aéroport choisira une grande compagnie qui a décidé de poser un avion en Europe du Sud ? Cette zone dépasse le cadre du Grand Sud Est de la France et nous pensons qu'une compagnie internationale se tournera certainement vers un aéroport d'une taille comprise entre 8 et 15 millions de passagers annuels. Tout ceci place la concurrence aéroportuaire, qui nous concernera, avec Barcelone, Milan, Munich et Zurich, puis, dans une moindre mesure, avec Genève et Lyon. A noter que Genève a un type de clientèle très proche de la notre. »

Un responsable de l'aéroport Marseille Provence, est plus radical sur ce thème de la complémentarité : "Nous sommes dans un environnement concurrentiel, notamment avec Lyon Saint-Exupéry et Nice Côte d'Azur, qui justifie le secret autour de notre stratégie réelle. "

Son homologue de l'aéroport de Lyon Saint-Exupéry confirme cette responsabilité stratégique fondée sur l'autonomie de fait des aéroports de province : « Nous ne croyons pas que l'Etat affichera un jour des directives dirigistes sur de possibles spécificités des aéroports... Le rôle de Lyon Saint-Exupéry est de développer l'offre de service transport pour les habitants et les entreprises de la région Rhône-Alpes dans un premier temps, du sud de l'Europe dans un 
second. L'aéroport doit être attentif aux besoins spécifiques des clients de la région. Pour cela il doit respecter les volontés politiques des élus locaux ».

On remarque donc que les responsables d'aéroport ne raisonnent en aucun cas à un niveau de solidarité élargi au Grand Sud-Est, sinon pour évoquer des services en correspondance. La concurrence est sous-entendue ou même franchement explicitée. Il ne s'agit pas tant d'une concurrence sur le marché des clientèles régionales que d'une concurrence vis à vis des compagnies aériennes dont il s'agit d'attirer les dessertes ou même un projet de hub.

\section{L’administration}

Les agents de l'administration interrogés appartiennent tous à l'Aviation civile. Tous placent le rôle de l'Etat à un niveau principalement technique : "Un rôle majeur de l'Etat est de réserver l'espace nécessaire à l'aménagement ou au développement des aéroports, ainsi que de protéger leur environnement (bruit, pollution ...)». "A travers l'Aviation civile, l'Etat contrôle et garantit que les compagnies respectent les normes de sécurité et que leurs moyens financiers sont suffisants. Par exemple, lorsqu'une collectivité ou une CCI subventionne une compagnie, l'Aviation civile contrôle que l'activité pourra durer sur l'ensemble de la période contractuelle (3 ans normalement) et impose des obligations de service public. Le rôle de l'Aviation civile est de mobiliser les moyens pour accompagner la demande des opérateurs ». Du point de vue économique, le respect des forces de marché est clairement annoncé : « Depuis deux ou trois gouvernements, l'Etat n'est plus directif sur le transport aérien. Il laisse les compagnies aériennes se développer comme elles l'entendent ».

La question des investissements en infrastructures est souvent développée : « L’Etat intervient très peu dans le financement des aménagements aéroportuaires régionaux. A titre d'exemple, lors du dernier agrandissement de l'aérogare de Clermont-Ferrand, le financement s'est réparti ainsi : Etat 20\%, Région 20\%, Département 20\%, Agglomération 20\%, Ville 20\%. De la même façon, à Marseille Provence, les collectivités n’ont apporté que 10\% du financement des derniers travaux, le reste venant d'emprunts et de fonds propres ». Le respect de cette autonomie d’investissement est fréquemment rappelé : "Les aéroports représentent des investissements beaucoup moins lourds que les lignes de trains. On peut préconiser de développer des aéroports, notamment dans des régions isolées, pour une durée limitée, 20 ans par exemple, en attendant que des infrastructures plus lourdes se justifient ».

S'il est une position qui a quelque importance politique, elle concerne le développement de petits aéroports qui pourrait être maîtrisée afin de favoriser l'essor des plus grands aéroports régionaux. L'administration n'est pas sur cette ligne, bien au contraire : « Il n'y a pas de raisons de freiner l'apparition ou le développement des aéroports de province, quelque soit leur taille. Ils sont toujours ardemment soutenus par leur CCI et les chefs d'entreprises locaux. Les aéroports de province sont indispensables pour le développement industriel et économique local et ils représentent des investissements tout à fait dans les moyens des CCI. En outre, on peut rappeler qu'ils sont comparativement fort peu coûteux. Un aéroport de taille modeste (type Béziers) peut être estimé au alentours de 10 millions d'Euros, une autoroute coûtera 30 à 100 millions d'Euros du kilomètre, et une ligne de TGV entre 10 et 200 millions d'Euros du kilomètre. De plus, ces aéroports pourront devenir une alternative à la saturation de ceux qui leurs sont proches ».

Ces échéances de saturations sont les événements les plus souvent évoqués lorsqu’il s’agit de trouver des complémentarités. A l'échelle des grands aéroports du Grand Sud-Est, il est 
généralement estimé que Nice devrait être saturé d’ici 10 ans. Lyon et Marseille le seront aussi à leur tour, dans un avenir rarement précisé. Il est alors imaginé un report par TGV entre ces aéroports, ou vers Montpellier, à mesure que chacun d'entre eux arrivera aux limites de ses capacités de développement. Il est aussi imaginé que de plus petits aéroports prennent le relais des premiers saturés, tel Avignon auprès de Marseille. Mais on remarque qu'aucune de ces perspectives n’est datée et moins encore planifiée.

\section{Les compagnies aériennes}

Les représentants des compagnies contactés ont peu d'avis sur la concurrence des aéroports. Bien que les compagnies aient parfois une activité privilégiée avec certains d'entre eux, rien ne les lie dans la durée : elles peuvent ouvrir ou fermer une ligne en quelques semaines. Le critère de rentabilité, s’il est absolu, n’est pas décliné de la même façon par toutes les compagnies. Certaines raisonnent effectivement en ligne, c'est par exemple, pour ce qui est du territoire français, le cas de la British Airways, comme le précise l'un de ses responsables : «Concernant les ouvertures de lignes, nous cherchons actuellement à densifier notre réseau. Mais tout projet d'ouverture de ligne doit être rentable à moyen-terme, soit environ 18 mois. C'est pour cela, par exemple, que nous n'avons pas ouvert pour l'instant de LondresStrasbourg, alors que Strasbourg est une ville importante en France. Néanmoins, cela fait l'objet d'une étude régulière tous les 6 mois. De la même façon, nous sommes prêts à fermer toute ligne qui n'est plus rentable ».

D’autres compagnies ont une politique de réseau. C’est notamment le cas d'Air France : «La principale préoccupation reste l'équilibre économique du réseau. Dans cette logique, il n'est pas question de renoncer aux liaisons difficiles qui contribuent à consolider l'économie d'autres liaisons. », explique un responsable des lignes «métropole » d'Air France, avouant toutefois les progrès encore attendus dans cette approche réseau du gestionnaire : " Dans toute analyse de la performance d'une ligne, les résultats « locaux » de la ligne sont complétés par une analyse de l'apport au réseau de cette ligne. Si nous ne disposons pas encore d'un outil permettant de mesurer l'impact de la modification d'une ligne (ou de son ouverture, ou de sa fermeture) sur l'ensemble du réseau, nous sommes cependant en mesure de prendre en compte le volume de correspondances apportées par une ligne et donc la marge supplémentaire dégagée par la ligne d’apport sur les autres lignes ».

Le même responsable évoque, cependant, deux expériences notables de spécialisation d'aéroports. Tout d'abord en Bretagne, où Air France a décidé de transférer d'Orly vers Roissy Charles de Gaulle la desserte parisienne de Lorient et de renforcer la desserte de Quimper vers Orly. « A Lorient, ..., l'aéroport n’en fut pas contrarié parce qu’il conservait la même masse atterrie et qu'il accédait au hub, mais nous avons observé une baisse de $4 \%$ du remplissage de Lorient-Paris ». Rappelons au passage, qu'en transport aérien, nombre de lignes ne vivent qu'à quelques points au dessus du taux de remplissage pivot.

L’autre expérience évoquée se situe dans le Grand Sud-Est: "Nîmes nous a demandé d'étudier la spécialisation : Nîmes-Roissy et Montpellier-Orly. Ceci aurait pour Air France comme conséquences d'offrir toute la clientèle internationale de et vers Montpellier à la concurrence ». Cet exemple suggère que le discours libéral généralement développé par les gestionnaires d'aéroports n'exclut pas certains réflexes d'administration de l'offre.

Plus largement, ces deux cas d'espèce montrent bien où se situe le pouvoir de décision qui détermine l'offre de transport. Les deux aéroports bretons ont subi une décision d'Air France 
et l'aéroport de Nîmes a été débouté dans sa requête. Les compagnies sont libres de rechercher l'offre la plus efficace sous les contraintes des marchés. Le discours d'un cadre supérieur d'Air France illustre volontairement cette rationalité par une hypothèse d'école pour le Grand Sud-Est : "Si Air France décidait de spécialiser Lyon sur Roissy et Grenoble sur Orly, il n'est pas certain que les hommes d'affaires lyonnais y auraient intérêt, et approuveraient la politique de la compagnie ». On peut même être certain du contraire. La seule réplique possible des aéroports serait, dans un tel cas, de trouver quelque opérateur acceptant de se lancer sur un segment de marché venant d’être délaissé par un autre.

\section{II.2. De faibles complémentarités dans les discours, comme dans les trafics}

Si les orientations stratégiques des uns et des autres sont assez claires, on observe que les acteurs ont chacun leurs bonnes raisons de récuser un objectif de complémentarité.

- Les concessionnaires d'aéroports interprètent cette notion de complémentarité comme une tentative d'organisation autoritaire de la part de l'Etat, qui pourrait contrarier leur autonomie et ferait peu de cas des tendances du marché. A contrario, prévaut un sentiment de concurrence entre les aéroports, qui est souvent explicité et dont témoignaient certaines réticences à se soumettre aux entretiens.

- Les administrations consultées ont bien compris cet état d'esprit, tout comme celui des compagnies aériennes, et souhaitent le respecter en accompagnant au mieux le développement de chacun des aéroports dans l'intérêt de la région ou des villes moyennes concernées. L'idée générale est que les usagers sont peu enclins à aller chercher un aéroport éloigné et qu'ils veulent un maximum de liaisons directes au départ de celui qui leur est proche.

- Les compagnies aériennes ironisent sur ce terme de complémentarité. Elles ont une très large autonomie pour choisir les lignes qu'elles exploitent et leurs critères sont soumis aux lois du marché. Elles choisissent l'aéroport et la ligne qui leur apporteront la meilleure rentabilité, celle-ci s'inscrivant dans un compromis entre concurrence (des autres compagnies ou du TGV) et capacité du marché local.

Cependant, cette récusation par les acteurs d'un raisonnement en termes de complémentarité, a fortiori de coordination, n’exclut pas que les stratégies de chacun ou les forces du marché déterminent des morphologies de réseau qui seraient finalement complémentaires de fait. Les spécificités révélées dans la partie précédente le suggèrent, telle l’orientation de Marseille Provence vers la Méditerranée. Mais entre spécificité et complémentarité, il y a une nuance : des complémentarités impliqueraient des échanges significatifs entre les aéroports eux-mêmes car ils se traduiraient par des rabattements réciproques de lignes. On pourrait, en effet imaginer que de telles liaisons ouvrent à la clientèle de l'un des quatre aéroports des destinations qui sont proposées par l'un des autres. La figure suivante (figure 5) confirme les discours des acteurs et nous montre qu'il n'en est rien. 


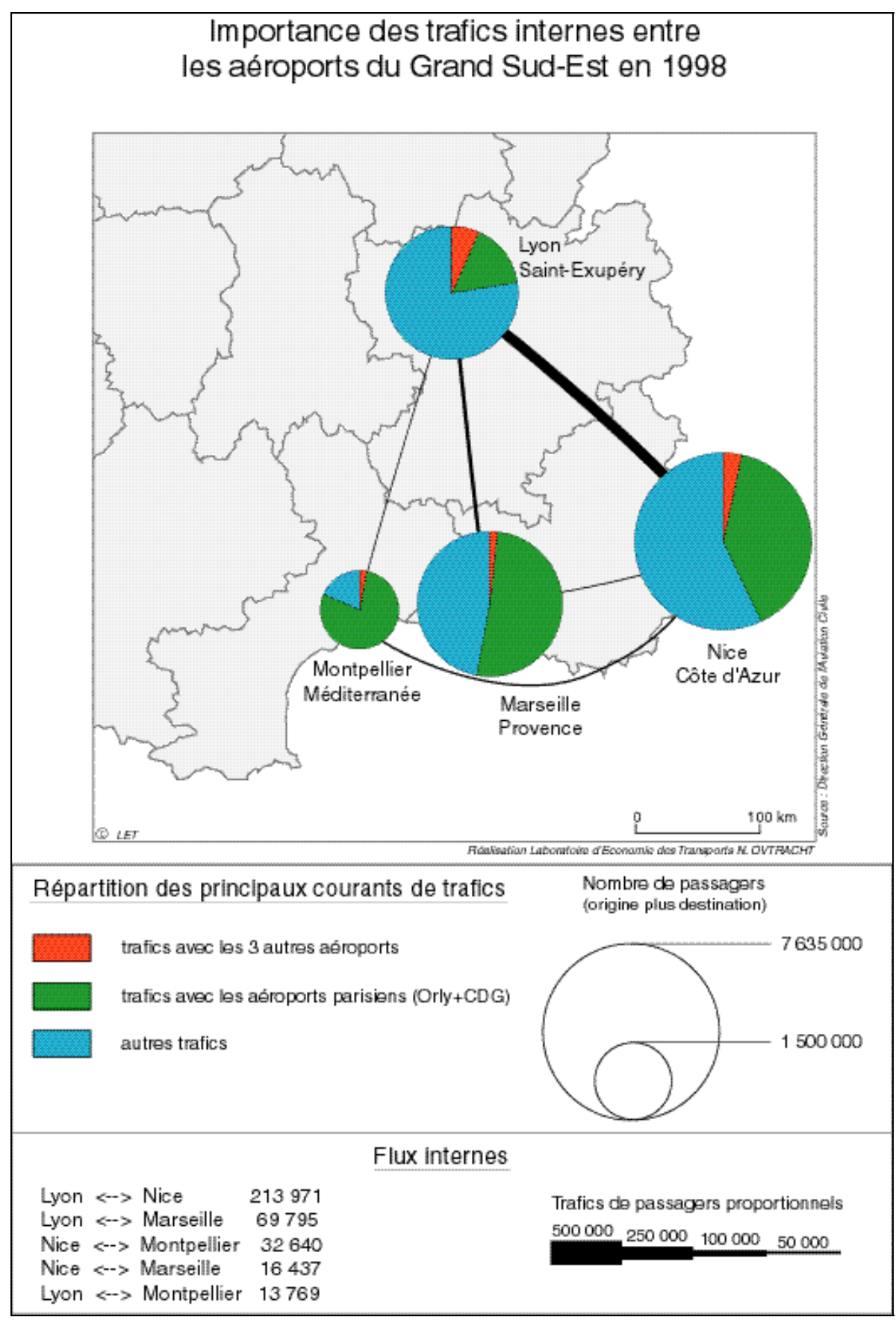

Figure 5 : Importance des trafics internes entre les aéroports du Grand Sud-Est en 1998

Seule la ligne Lyon - Nice a une importance significative. Nous avons cependant observé que les destinations offertes dans ces deux aéroports se recouvraient largement, sauf pour quelques destinations en province à partir de Lyon, qui peuvent intéresser la clientèle niçoise, et quelques destinations de Nice vers l'Europe du Nord. Pour ces dernières la clientèle lyonnaise ne peut guère être attirée avec un tel détour par Nice.

La ligne Lyon - Marseille n'avait pas encore subi en 1998 la concurrence du TGV Méditerranée et son affaiblissement, depuis, la situe d'autant plus mal pour rabattre la clientèle marseillaise vers Saint-Exupéry que ce TGV passe par cet aéroport ${ }^{8}$. En revanche, de

\footnotetext{
8 Les horaires de TGV s'arrêtant à Lyon Saint-Exupéry ne sont pas, aujourd'hui, favorables aux correspondances, mais on peut penser qu'ils le deviendront progressivement, comme cela s'est réalisé pour la gare TGV de Roissy Charles de Gaulle.
} 
Lyon vers Marseille, un bon système de correspondance vers le Sud devrait correspondre à une bien meilleure demande.

Le cas de Montpellier Méditerranée est illustratif de ces faibles complémentarités : l’aéroport où il $\mathrm{y}$ a le moins de destinations directes offertes n'est pas spécialement tourné vers les aéroports de la région pour y profiter d'éventuelles correspondances. Au demeurant, la ligne Montpellier - Lyon a été fermée courant 1998. Paris semble rester la référence pour aller chercher une correspondance. En effet, Londres, seconde ligne au départ de Montpellier Méditerranée, ne « pèse » que $6 \%$ de la ligne avec Paris.

Du reste, un rapide examen des exemples de complémentarité organisée de par le monde ${ }^{9}$ explique assez bien que le Grand Sud-Est se prête mal à ce type d'organisation. Il existe, en effet, quelques cas de systèmes aéroportuaires mais peu sont à l'échelle régionale et tous sont à l'initiative de compagnies aériennes.

\section{II.3. Quelques exemples de complémentarités aéroportuaires dans le monde}

Tout d'abord, il y a le cas de la plupart des grandes métropoles qui sont desservies par plusieurs aéroports. On observe alors généralement que la compagnie nationale les utilisent tous au bénéfice de sa logique de réseau. Ainsi, British Airways a pu trouver à Gatwick des ressources devenues rares à Heathrow ; Air France a réservé à Orly un rôle d'accès provincecapitale pour mieux exploiter à Roissy un hub international. Mais il s'agit chaque fois de logiques “internes", ces aéroports étant gérés par une autorité unique et la compagnie nationale ayant, de longue date, bénéficié d'une protection du pouvoir central.

Dans quelques cas, les compagnies vont chercher au loin un second aéroport majeur, selon des logiques diverses. En Allemagne, Lufthansa tend à spécialiser ses deux hubs : à Francfort le trafic à haute contribution de l'international, à Munich le reliquat. Ce qui ne l'a pas empêchée de s'investir pleinement dans une expérience unique en Europe. Lufthansa a cofinancé et cogérera avec l'autorité aéroportuaire locale, le terminal 2 de Munich qui ouvrira à l'été 2003. Pour cela la compagnie détient $40 \%$ de la joint-venture qui les unit.

Air France, en 1997, a arrêté le principe d'un hub secondaire à Lyon. Elle y perpétue la tradition d’Air Inter du "rendez-vous » national déjà évoqué et y développe un trafic moyencourrier européen dont toutes les destinations sont également desservies depuis Paris. Les raisons réelles de cette stratégie demeurent ambiguës alors que le hub central est loin d'être saturé. D’aucuns ont évoqué une sorte de jeu de go qui consiste à occuper une plate-forme que pourraient convoiter d'autres grands opérateurs. Ce hub et les lignes qui la composent sont de plus en plus rentables, mais cela ne permet pas de conclure que Lyon doive jouer un rôle stratégique dans le développement de la compagnie.

Alitalia est à l'origine d'une organisation en double hub, particulièrement rare en Europe. Avec son "Systema Italia”, elle a choisi depuis peu de distribuer son réseau autour de deux points centraux que sont Milan-Malpensa et Rome-Fiumicino. Pour éviter les redondances, Rome, hub d'origine, a perdu quelques destinations directes au profit de Milan, où Alitalia veut enrayer l'évasion des hommes d'affaires vers les hubs de ses concurrents du Nord. A Fiumicino est dévolu l'accès à l'Italie méridionale et demeure la desserte de l'Amérique latine, l'Extrême Orient et l'Afrique. A Malpensa sont privilégiées l'Italie du Nord et l'Amérique du Nord. Entre les deux, de nombreux vols font le "hub to hub". Nous sommes bien, en ce cas, face à un choix délibéré de complémentarité, mais qui n’était possible que

\footnotetext{
${ }^{9}$ Aéroports Magazine, octobre et novembre 2000, propose une présentation complète de ces expériences.
} 
parce que la même compagnie avait une position dominante à Rome et qu'une nouvelle plateforme était créée à Milan.

En France, Air Littoral a eu une organisation similaire entre 1996 et 2000, à un niveau évidemment plus modeste, avec un hub Nord-Sud à Montpellier et un hub Est-Ouest à Nice, tous deux reliés par des services adaptés aux correspondances. Cela ne signifie pas pour autant qu'il y avait coopération entre ces deux aéroports. Pour Nice, du reste, Air Littoral ne représentait en 1997 que 8\% de son trafic.

Si la coopération entre aéroports régionaux est rarissime, on observe cependant de plus en plus de cas de prise de participation financières entre aéroports ou de concessions d'exploitation multiples, reposant sur la tendance à la privatisation. L'aéroport de Vienne a gagné l'exploitation du futur aéroport de Berlin. La British Airport Authority, privatisée en 1986, exploite 7 plates-formes au Royaume-Uni, contrôle Naples et Melbourne depuis 1997 et a des intérêts outre-Atlantique à Newark, Pittsburgh et Indianapolis. L'Aena, qui gère 37 aéroports espagnols, est un des leaders du consortium qui a remporté le contrat de concession de 12 aéroports mexicains. La SEA italienne, en charge des deux aéroports de Milan, est actionnaire à 28\% de la société responsable de 33 aéroports argentins. Aer Rianta (Dublin, Shannon, Cork) a des intérêts à Birmingham et Dusseldorf. Francfort a construit, en association avec un groupe de BTP, le nouvel aéroport d'Athène-Spata et en assurera l'exploitation. Le groupe ADP possède des participations dans des aéroports africains et en a pris récemment de nouvelles à Liège, Pékin et au Mexique (autre consortium concessionnaire de 13 aéroports).

Outre que ces participations se font souvent d'un pays à l'autre, elles témoignent de la part de grands gestionnaires nationaux, d'une course à la taille mondiale. Il s'agit de stratégies de développement dans un métier, la gestion des aéroports, qui n’implique pas une influence directe sur la politique des compagnies.

Qu'il s'agisse du rôle dominant des compagnies, des configurations à l'Italienne qui justifient le choix de la complémentarité ou des politiques des aéroports, rien ne nous autorise à imaginer qu'une complémentarité puisse être délibérément coordonnée au niveau du Grand Sud-Est.

\section{II.4. Un contexte confirmé de régulation concurrentielle}

Les évolutions récentes vont dans le sens d'une plus grande responsabilisation des autorités aéroportuaires. En octobre 2000, au $10^{\text {ème }}$ congrès annuel de l'Alfa-ACI ${ }^{10}$, Hervé de Place, Président de l'Alfa-ACI et directeur général de l'aéroport de Nice, expliquait : « Sous l'égide de quelques grandes CCI membres de l'Uccega ${ }^{11}$, nous avons présenté aux pouvoirs publics un texte qui précise notre projet d'évolution du cadre réglementaire des aéroports. L’objectif de ce projet consiste à donner à l'industrie aéroportuaire française les moyens de rester compétitive face aux aéroports européens leaders en plein processus de privatisation et de création d'alliances capitalistiques ». Cette déclaration est liée à deux points importants dans les stratégies des aéroports régionaux.

\footnotetext{
${ }^{10}$ Association des aéroports de langue française associée à l’ACI.

${ }^{11}$ Union des chambres de commerce et gestionnaires d'aéroports.
} 
En premier lieu, c'est dans un contexte global de grandes manœuvres financières et de concurrence généralisée pour le choix des gestionnaires d'aéroport que s’inscrira à l'avenir la stratégie des aéroports français, ainsi que l’illustrent quelques uns des exemples évoqués au précédent paragraphe.

En second lieu, le renouvellement pour bon nombre d'entre eux des contrats de concession témoigne des nouvelles orientations publiques en la matière. André Tanti, du ministère des finances, rappelle que «l'Etat ne veut plus prendre le risque de miser sur les aéroports régionaux. L'Etat se désengage des petites plates-formes », ce que confirme Jean-Pierre Cahingt, membre du comité directeur de l’Uccega et vice-président de la CCI de Nantes : « il faut que la communauté aéroportuaire soit extrêmement vigilante face aux nouvelles formes de concession aéroportuaire. Dans les textes, il est en effet expressément stipulé que les gestionnaires d'aéroports s'engagent désormais “à leurs risques et périls” et, de plus, sur des périodes relativement courtes, de l'ordre de 3 à 5 ans ».

Le risque n'est plus alors couvert par les finances publiques. Tous les acteurs gardent probablement en mémoire l'expérience malheureuse de Saint-Etienne Bouthéon, où les collectivités locales ont investi 26,5 millions de francs en rénovation des installations aéroportuaires pour accueillir le hub de Proteus. En l'espace de 26 mois, cette compagnie a installé progressivement 12 lignes quotidiennes puis, peu à peu, les a intégralement retirées. Les optimistes se consolent avec l'arrivée de la compagnie à bas coûts Ryanair qui a limité le déclin du trafic grâce à un gros tronçon vers Stansted : d'un pic de 195.000 passagers en 1998, l'aéroport est passé en 2000 à 120.000 passagers. De telles aventures seront de moins en moins fréquentes à mesure que la seule logique marchande s’imposera dans ce secteur.

Il reste que des aéroports modestes peuvent capturer des trafics et entraver le développement de plus grands. Jacques Pavaux, directeur de l'ITA ${ }^{12}$, en donne des exemple dont l'un concerne le Grand Sud-Est : «Avec Ryanair, Carcassonne prend du trafic à Montpellier et Toulouse, Dinard en prend à Rennes... et Beauvais à Paris ». Ce qui n’est pas forcément néfaste pour Paris, peut-être plus critique pour Montpellier qui se bat déjà contre des rivaux plus grand qu'elle : selon une étude de l'ITA, 52\% seulement des passagers locaux prennent l'avion à Montpellier-Méditerranée, les autres se partageant entre Marseille et Lyon.

On remarque donc, à la lumière de ces témoignages, que la concurrence entre les aéroports de province est une réalité qui se confirme. Mais une concurrence à double niveau : entre platesformes régionales en quête de masse critique cherchant à attirer des compagnies et entre ces plates-formes et les aéroports secondaires cherchant à attirer les mêmes passagers.

\section{Conclusion : des stratégies autonomes}

Aucun des quatre principaux aéroports du Grand Sud-Est n’a de vraie raison d'infléchir la stratégie de développement qu’il a élaborée au cours des années 1990.

Lyon Saint-Exupéry, fort d'avoir été «choisi » par Air France comme second hub possède deux atouts importants, l'embranchement TGV qui ne demande qu'à bénéficier d'arrets plus favorables aux correspondances et d'importantes réserves foncières. Seuls les riverains, peu nombreux mais très actifs, pourraient être un facteur limitant le développement, mais la prise en compte de ce phénomène semble être conduite efficacement.

\footnotetext{
${ }^{12}$ Institut du Transport Aérien.
} 
Marseille Provence a pour ambition majeure d'être la plate-forme de correspondance des trafics nord-sud transméditerranéens à destination de la Corse, du Maghreb, de l'Afrique Subsaharienne et de l'océan indien. Air France, dès le programme 1999, a commencé à développer ses services dans ce sens.

Montpellier Méditerranée s'est donné pour objectif de devenir une "plate-forme principale de désenclavement du bassin méditerranéen" qui relierait l'Espagne et l'Italie, le nord et le sud de l'Europe. Il prévoit de relayer Marseille, Nice ou Toulouse lorsqu'ils seront arrivés à saturation. Cette ambition paraît techniquement possible dans la mesure où Montpellier Méditerranée est très loin d'être saturé et possède toutes les réserves foncières dont il pourrait avoir besoin à ces fins. Mais il s'agit tout de même de perspectives de long-terme. Air Littoral a donné à ce dessein une première réalité à la mesure de sa taille et montré que cette orientation stratégique n’est peut être pas complètement utopique.

Nice Côte d'Azur souhaite devenir un des premiers hubs secondaires européens. Ses trafics sont très dynamiques et bien diversifiés vers l'Europe. Ses dirigeants y mènent de grandes actions marketing pour attirer de nouveaux voyageurs, notamment italiens et de nouvelles compagnies aériennes. Mais ce succès risque de très vite buter sur les limites physiques de capacité de l'aéroport car il n'y a plus guère de perspectives d'agrandissement à l'issue des travaux actuels.

Attirer des compagnies et de nouvelles lignes. Profiter au mieux des héritages de l'histoire, en particulier des relations privilégiées avec certaines destinations. Accéder à la catégorie supérieure pour changer de position dans le regard des compagnies. Au total favoriser la diversification des services offerts pour mieux accéder au reste du monde. Il n’y a rien là que des ambitions et des moyens légitimes de la part des aéroports, mais qui sont surplombés par un jeu concurrentiel des compagnies aériennes qui laisse peu de place à une complémentarité organisée.

\section{Bibliographie}

ARABEYRE A., BONNAFOUS A. (dir.), GIRET A, OVTRACHT N., 2000, Les perspectives d'évolution des aéroports du Grand Sud-Est de la France: quelles complémentarités ? (phase 2), Laboratoire d'Economie des Transports, 42 p.

BONNAFOUS A. (dir.), GIRET A, 1999, Les perspectives d'évolution des aéroports du Grand Sud-Est de la France : état des lieux et stratégies d'acteurs. (phase 1), Laboratoire d'Economie des Transports, 178 p.

CARRE A-D., 2000, Aéroports et stratégies d'entreprises, Presses de l'ITA, 2 vol. 726 et 388 p.

COLLIN M. (dir.), 2000, Aéroports et dynamiques des territoires, Rapport pour le PREDIT, $184 \mathrm{p}$.

DATAR, 2000, Aménager la France de 2020. Mettre les territoires en mouvement, La documentation française, $87 \mathrm{p}$.

Direction de l'Aviation Civile (DAC) Sud-Est, Résultats généraux du trafic commercial des aérodromes et des lignes aériennes, année 1997, Département Programme, 18 p. 
Direction Générale de l'Aviation Civile (DGAC), juillet 1995, Vers un Schéma Directeur des Infrastructures Aéroportuaires. Avec la contribution des bureaux d'études BIPE et ITA.

DORMOY F, 1997, La concurrence entre aéroports, quelques exemples en Europe, 10è Entretiens Jacques Cartier, 8-9 décembre 1997, Lyon.

HUAULT P., 1997, Evolution du transport aérien en Europe de 1992 à 1996, Notes de synthèses du SES, novembre décembre, 6 p.

Institut du Transport Aérien (ITA), 1999, Tableau de bord du transport aérien dans le monde, Données 1997, Etudes \& Documents, vol. 47, 110 p.

MALDUTIS J., MUSANTE R., 1993, Airline competition at the 50 largest US Airports, Salomon Brothers, New-York.

MOLIN B., 1997, Eléments d'économie des réseaux "Hub and spokes" aériens. Expérience américaine, perspectives. Thèse de doctorat en économie, Laboratoire d'Economie des Transports, université Lumière-Lyon 2, 365 p.

MOLIN B., 1998, Eléments pour une prospective du transport aérien européen, DATAR programme de recherche prospective 1996/2000, rapport final avril 1998, 60 p.

PAVAUX J., 1984, L'économie du transport aérien. La concurrence impraticable, Economica, 434 p.

PAVAUX J. (dir.), 1995, Le transport aérien à l'horizon 2020 : éléments de réflexion prospective, Presses de l'ITA, PUF, 234 p. 